\title{
Dirac Operators on Noncommutative Curved Spacetimes ${ }^{\star}$
}

\author{
Alexander SCHENKEL ${ }^{\dagger}$ and Christoph F. UHLEMANN $\ddagger$ \\ $\dagger$ Fachgruppe Mathematik, Bergische Universität Wuppertal, \\ Gaußstraße 20, 42119 Wuppertal, Germany \\ E-mail: schenkel@math.uni-wuppertal.de \\ $\ddagger$ Department of Physics, University of Washington, Seattle, WA 98195-1560, USA \\ E-mail: uhlemann@uw.edu
}

Received August 09, 2013, in final form December 11, 2013; Published online December 15, 2013 http://dx.doi.org/10.3842/SIGMA.2013.080

\begin{abstract}
We study the notion of a Dirac operator in the framework of twist-deformed noncommutative geometry. We provide a number of well-motivated candidate constructions and propose a minimal set of axioms that a noncommutative Dirac operator should satisfy. These criteria turn out to be restrictive, but they do not fix a unique construction: two of our operators generally satisfy the axioms, and we provide an explicit example where they are inequivalent. For highly symmetric spacetimes with Drinfeld twists constructed from sufficiently many Killing vector fields, all of our operators coincide. For general noncommutative curved spacetimes we find that demanding formal self-adjointness as an additional condition singles out a preferred choice among our candidates. Based on this noncommutative Dirac operator we construct a quantum field theory of Dirac fields. In the last part we study noncommutative Dirac operators on deformed Minkowski and AdS spacetimes as explicit examples.
\end{abstract}

Key words: Dirac operators; Dirac fields; Drinfeld twists; deformation quantization; noncommutative quantum field theory; quantum field theory on curved spacetimes

2010 Mathematics Subject Classification: 81T75; 81T20; 83C65

\section{Introduction and summary}

Noncommutative geometry has long been of interest from a purely mathematical perspective as a natural generalization of ordinary differential geometry. It is also of crucial interest from a physical perspective, since it generically plays a role when the principles of quantum mechanics are combined with those of general relativity $[17,18]$. In both contexts, Dirac operators are of major importance: they are relevant for structural questions in noncommutative geometry [13] and essential for the description of fermionic fields in models for high-energy physics. In this article we focus on the latter point and study Dirac operators as equation of motion operators for Dirac fields on noncommutative spacetimes. The noncommutative geometries which we are going to consider are obtained by formal deformation quantization of smooth manifolds via Abelian Drinfeld twists. Since we are interested in generic curved spacetimes, we will not assume compatibility conditions between the geometry and the twist. In particular, we do not restrict ourselves to twists generated solely by Killing vector fields, as these are just not available on generic spacetimes.

The construction of Dirac operators in our generic setting turns out to be much more involved than in the highly symmetric setup with twists constructed solely from Killing vector fields. As shown in [14], the classical Dirac operator is in this special case also a valid Dirac operator on the

\footnotetext{
${ }^{\star}$ This paper is a contribution to the Special Issue on Deformations of Space-Time and its Symmetries. The full collection is available at http://www.emis.de/journals/SIGMA/space-time.html
} 
noncommutative manifold. In the generic case, however, there are several natural deformations of the classical Dirac operator, which at first seem equally well motivated and are not obviously equivalent. It is thus not clear which operator we should choose, and that state of affairs certainly is not satisfactory. To improve on it, we will propose an abstract characterization of a Dirac operator on noncommutative curved spacetimes, in terms of a minimal set of axioms. Namely, it should be a differential operator of first order in a sense appropriate for noncommutative geometry, it should be constructed from geometric objects like the spin connection and the vielbein alone, and it should have the correct classical limit. We can then study to which extent the various explicit constructions realize these properties, and under which circumstances they turn out to be equivalent.

We find that the minimal set of axioms does in general not uniquely select one of the constructions that we are going to present. That is, we show that two of our operators satisfy the axioms and turn out to be inequivalent. As is to be expected, the classical Dirac operator is not among them. Restricting then to more special classes of deformations, we find that for twists constructed from sufficiently many Killing vector fields (semi-Killing twists, which are important for studying solutions of the noncommutative Einstein equations [2, 26, 33]), our candidates for Dirac operators all agree and fit into our general characterization of noncommutative Dirac operators. The freedom in choosing a Dirac operator is thus reduced drastically in this special class of deformations. Furthermore, as one is thus free to choose the technically most convenient definition, this can simplify explicit calculations considerably. The classical Dirac operator meets our axioms for noncommutative Dirac operators only when restricting to actual Killing twists, and it then coincides with all the deformed constructions. Turning back to the general case of twists which do not necessarily involve Killing vector fields, the question remains which of the (e.g. inequivalent) noncommutative Dirac operators one should prefer. Having in mind the construction of noncommutative quantum field theories, it is natural to also demand formal self-adjointness with respect to a suitable inner product. We will find that this requirement indeed singles out a preferred choice among our candidates, and we will outline the construction of a quantum field theory of noncommutative Dirac fields. A natural next step would be to aim for a complete classification of noncommutative Dirac operators satisfying our axioms, which we leave for future work.

The outline of this paper is as follows: For the coupling of Dirac fields to the noncommutative background geometry we employ techniques of twist-deformed noncommutative geometry and noncommutative vielbein gravity [1], which we review in Section 2. In Section 3 we discuss three well-motivated deformations of the classical Dirac operator, and present our minimal set of axioms for Dirac operators in the noncommutative setting. We then show that two of the proposed operators generally satisfy these axioms. In Section 4 we show that for generic noncommutative curved spacetimes the two noncommutative Dirac operators are inequivalent, and that adding formal self-adjointness as an additional condition selects a unique Dirac operator, at least among the examples we have provided. Furthermore, restricting to a special class of deformations given by semi-Killing twists we show that the ambiguities in defining noncommutative Dirac operators disappear in these highly symmetric models, where the twist contains sufficiently many Killing vector fields. In Section 5 we outline the construction of noncommutative Dirac quantum field theories. To illustrate our constructions we provide in Section 6 explicit examples and formulas for noncommutative Dirac operators on spacetimes of physical interest.

\section{Preliminaries}

In the following we review techniques from deformation quantization of smooth manifolds by Abelian Drinfeld twists and the framework of noncommutative vielbein gravity, as far as they will be relevant for the main part. 


\section{$2.1 \quad$ Twist-deformed noncommutative geometry}

Let $M$ be a $D$-dimensional manifold. The noncommutative geometries that we shall consider are those which arise as deformations of $M$ by an Abelian Drinfeld twist

$$
\mathcal{F}:=e^{-\frac{i \lambda}{2} \Theta^{\alpha \beta} X_{\alpha} \otimes X_{\beta}},
$$

where $\Theta^{\alpha \beta}$ is an antisymmetric, real and constant matrix (not necessarily of rank $D$ ) and $X_{\alpha}$ are mutually commuting real vector fields on $M$, i.e. $\left[X_{\alpha}, X_{\beta}\right]=0$ for all $\alpha, \beta .{ }^{1}$ The deformation parameter $\lambda$ is assumed to be infinitesimally small, i.e. we work in formal deformation quantization. In this setup a formal power series extension $\mathbb{C}[[\lambda]]$ of the complex numbers, as well as of all vector spaces, algebras, etc., has to be performed, but for notational simplicity we will suppress the square brackets $[[\lambda]]$ denoting these extensions. We can assume, without loss of generality, that $\Theta^{\alpha \beta}$ is of the canonical (Darboux) form

$$
\Theta=\left(\begin{array}{ccccc}
0 & 1 & 0 & 0 & \ldots \\
-1 & 0 & 0 & 0 & \ldots \\
0 & 0 & 0 & 1 & \ldots \\
0 & 0 & -1 & 0 & \ldots \\
\vdots & \vdots & \vdots & \vdots & \ddots
\end{array}\right) .
$$

It is convenient to introduce the following notation.

Definition 2.1. A twisted manifold is a pair $(M, \mathcal{F})$, where $M$ is a $D$-dimensional manifold and $\mathcal{F}$ is an Abelian Drinfeld twist, cf. (2.1).

On any twisted manifold $(M, \mathcal{F})$ we can develop a canonical noncommutative differential geometry. As a first step, consider $C^{\infty}(M)$, the space of all complex-valued smooth functions on $M$. By using the twist $\mathcal{F}$, we can equip this space with an associative and noncommutative product (the $\star$-product)

$$
f \star g:=\mu\left(\mathcal{F}^{-1} f \otimes g\right)=f g+\frac{i \lambda}{2} \Theta^{\alpha \beta} X_{\alpha}(f) X_{\beta}(g)+\cdots,
$$

where $\mu$ is the usual point-wise product and the action of the vector fields $X_{\alpha}$ on the functions $f, g$ is via the (Lie) derivative.

Moreover, the de Rham calculus $\left(\Omega^{\bullet}(M):=\bigoplus_{n=0}^{D} \Omega^{n}(M), \wedge, \mathrm{d}\right)$ on $M$ can be deformed by $\mathcal{F}$ into a differential calculus on the $\star$-product algebra $\left(C^{\infty}(M), \star\right)$. Explicitly, we define the $\wedge_{\star}$-product

$$
\omega \wedge_{\star} \tau:=\wedge\left(\mathcal{F}^{-1} \omega \otimes \tau\right)=\omega \wedge \tau+\frac{i \lambda}{2} \Theta^{\alpha \beta} \mathcal{L}_{X_{\alpha}}(\omega) \wedge \mathcal{L}_{X_{\beta}}(\tau)+\cdots,
$$

where the action of the vector fields $X_{\alpha}$ on the differential forms $\omega, \tau$ is via the Lie derivative. The undeformed differential $d$ satisfies the graded Leibniz rule with respect to the $\wedge_{\star}-$ product, i.e. $\mathrm{d}\left(\omega \wedge_{\star} \tau\right)=(\mathrm{d} \omega) \wedge_{\star} \tau+(-1)^{|\omega|} \omega \wedge_{\star}(\mathrm{d} \tau)$ with $|\omega|$ denoting the degree of $\omega$, and hence $\left(\Omega^{\bullet}(M), \wedge_{\star}, \mathrm{d}\right)$ is a differential calculus over $\left(C^{\infty}(M), \star\right)$. We extend the involution $*$ on $\left(C^{\infty}(M), \star\right)$, which is given by point-wise complex conjugation, to a graded involution on $\left(\Omega^{\bullet}(M), \wedge_{\star}, \mathrm{d}\right)$ by applying the rules $\left(\omega \wedge_{\star} \tau\right)^{*}=(-1)^{|\omega|}|\tau| \tau^{*} \wedge_{\star} \omega^{*}$ and $(\mathrm{d} \omega)^{*}=\mathrm{d}\left(\omega^{*}\right)$. The undeformed integral $\int_{M}: \Omega^{D}(M) \rightarrow \mathbb{C}$ satisfies the graded cyclicity property. This means that, for all $\omega, \tau \in \Omega^{\bullet}(M)$ with compact overlapping support and such that $|\omega|+|\tau|=D$,

$$
\int_{M} \omega \wedge_{\star} \tau=\int_{M} \omega \wedge \tau=(-1)^{|\omega||\tau|} \int_{M} \tau \wedge_{\star} \omega
$$

\footnotetext{
${ }^{1}$ The reason for restricting to Abelian Drinfeld twists is the validity of the graded cyclicity property (2.3), which does not hold true for generic Drinfeld twists.
} 
Finally, we deform the contraction operator $\iota$ (interior product) between vector fields and one-forms on $M$ by the twist $\mathcal{F}$. The resulting $\star$-contraction operator $\iota^{\star}$ is given by (cf. [4])

$$
\iota_{v}^{\star}(\omega):=\iota\left(\mathcal{F}^{-1} v \otimes \omega\right)=\iota_{v}(\omega)+\frac{i \lambda}{2} \Theta^{\alpha \beta} \iota_{\mathcal{L}_{X_{\alpha}}(v)}\left(\mathcal{L}_{X_{\beta}}(\omega)\right)+\cdots,
$$

where again the vector fields $X_{\alpha}$ act via the Lie derivative on vector fields $v$ and one-forms $\omega$.

This completes our snapshot review of twist-deformed noncommutative geometry and we refer the reader to [29] for a more detailed discussion.

\subsection{Noncommutative vielbein gravity}

The explicit constructions and examples of the main part will involve spacetimes of dimension 2 and 4 , and we therefore give the formalism of noncommutative vielbein gravity for both cases in the following. Throughout, $(M, \mathcal{F})$ will denote a twisted manifold of appropriate dimension. Following [1], we describe the noncommutative gravitational field by a noncommutative vielbein field $V$ and a noncommutative spin connection $\Omega$. Both are Clifford-algebra valued one-forms. Our gamma-matrix conventions are collected for easy reference in Appendix A. We say that the noncommutative spin connection is $\star$-torsion free if $0=\mathrm{d}_{\Omega} V:=\mathrm{d} V-\{\Omega \star V\}$, where $\{\Omega \stackrel{\star}{,} V\}=\Omega \wedge_{\star} V+V \wedge_{\star} \Omega$ is the $\star$-anticommutator. The $\star$-torsion constraint is part of the equations of motion of noncommutative vielbein gravity [1]. For reasons of generality, we do not assume the $\star$-torsion constraint for our general constructions and we shall clearly indicate at which later step it is used.

$\boldsymbol{D}=4$. We can expand $V$ and $\Omega$ in terms of the gamma-matrix basis $\left\{1, \gamma_{5}, \gamma_{a}, \gamma_{a} \gamma_{5}, \gamma_{a b}\right\}$ of the 4-dimensional Clifford algebra as

$$
V=V^{a} \gamma_{a}+\tilde{V}^{a} \gamma_{a} \gamma_{5}, \quad \Omega=\frac{1}{4} \omega^{a b} \gamma_{a b}+i \omega 1+\tilde{\omega} \gamma_{5}
$$

We further demand the reality conditions $V^{\dagger}=\gamma_{0} V \gamma_{0}$ and $\Omega^{\dagger}=-\gamma_{0} \Omega \gamma_{0}$. Notice that noncommutative $D=4$ vielbein gravity contains more fields than its commutative counterpart, where $\tilde{V}^{a}=\omega=\tilde{\omega}=0$. The reason is that $\mathrm{SL}(2, \mathbb{C})$ (Lorentz) $\star$-gauge transformations do not close and have to be extended to $\mathrm{GL}(2, \mathbb{C}) \star$-gauge transformations. The $\star$-gauge transformations act on $V$ and $\Omega$ by

$$
\delta_{\epsilon} V=\left[\epsilon^{\star}, V\right], \quad \delta_{\epsilon} \Omega=\mathrm{d} \epsilon+\left[\epsilon^{\star}, \Omega\right],
$$

where $\epsilon=\frac{1}{4} \epsilon^{a b} \gamma_{a b}+i \varepsilon 1+\tilde{\varepsilon} \gamma_{5}$ is a Clifford algebra valued function and $\left[\epsilon^{\star}, V\right]:=\epsilon \star V-V \star \epsilon$ is the $\star$-commutator. We impose the reality condition $\epsilon^{\dagger}=-\gamma_{0} \epsilon \gamma_{0}$.

As in [1] we require that $\left.\tilde{V}^{a}\right|_{\lambda=0}=\left.\omega\right|_{\lambda=0}=\left.\tilde{\omega}\right|_{\lambda=0}=0$, such that the commutative limit yields a usual commutative $\mathrm{SL}(2, \mathbb{C})$ vierbein and spin connection. We shall use the notation $\left(V_{(0)}, \Omega_{(0)}\right):=\left(\left.V\right|_{\lambda=0},\left.\Omega\right|_{\lambda=0}\right)$.

Definition 2.2. Let $(M, \mathcal{F})$ be a 4-dimensional twisted manifold. A noncommutative Cartan geometry on $(M, \mathcal{F})$ is a pair of Clifford algebra valued one-forms $(V, \Omega)$, satisfying the expansion (2.5), the reality conditions $V^{\dagger}=\gamma_{0} V \gamma_{0}, \Omega^{\dagger}=-\gamma_{0} \Omega \gamma_{0}$ and the limit $\left.\tilde{V}^{a}\right|_{\lambda=0}=\left.\omega\right|_{\lambda=0}=$ $\left.\tilde{\omega}\right|_{\lambda=0}=0$.

Let us now consider Dirac fields, i.e. functions $\psi \in C^{\infty}\left(M, \mathbb{C}^{4}\right)$ valued in the fundamental representation of the Clifford algebra. We denote the Dirac adjoint by $\bar{\psi}:=\psi^{\dagger} \gamma_{0}$. The $\star$-gauge transformations act on $\psi$ and $\bar{\psi}$ by $\delta_{\epsilon} \psi=\epsilon \star \psi$ and $\delta_{\epsilon} \bar{\psi}=-\bar{\psi} \star \epsilon$, respectively. Notice that the matrix $\psi \star \bar{\psi}$ transforms in the adjoint representation, $\delta_{\epsilon}(\psi \star \bar{\psi})=[\epsilon,, \psi \star \bar{\psi}]$. For all Dirac fields $\psi_{1}, \psi_{2}$ with compact overlapping support we define the inner product

$$
\left\langle\psi_{1}, \psi_{2}\right\rangle:=i \int_{M} \operatorname{Tr}\left(\psi_{2} \star \overline{\psi_{1}} \star V \wedge_{\star} V \wedge_{\star} V \wedge_{\star} V \gamma_{5}\right),
$$

which is $\star$-gauge invariant due to $(2.3),(2.6)$ and the cyclicity of the matrix trace Tr. 
Lemma 2.3. Let $(V, \Omega)$ be a noncommutative Cartan geometry on a 4-dimensional twisted manifold $(M, \mathcal{F})$. Then the inner product $(2.7)$ is hermitian, it reduces to the canonical commutative one for $\lambda=0$ and it is non-degenerate, i.e.:

a) $\left\langle\psi_{1}, \psi_{2}\right\rangle^{*}=\left\langle\psi_{2}, \psi_{1}\right\rangle$.

b) $\left\langle\psi_{1}, \psi_{2}\right\rangle=\int_{M} \overline{\psi_{1}} \psi_{2} \operatorname{vol}_{(0)}+\mathcal{O}(\lambda)$, where $\operatorname{vol}_{(0)}=V_{(0)}^{a} \wedge V_{(0)}^{b} \wedge V_{(0)}^{c} \wedge V_{(0)}^{d} \epsilon_{a b c d}$.

c) If $\left\langle\psi_{1}, \psi_{2}\right\rangle=0$ for all $\psi_{2}$, then $\psi_{1}=0$.

Proof. We show a) by the following short calculation

$$
\begin{aligned}
\left\langle\psi_{1}, \psi_{2}\right\rangle^{*} & =-i \int_{M} \operatorname{Tr}\left(\gamma_{5}^{\dagger} V^{\dagger} \wedge_{\star} V^{\dagger} \wedge_{\star} V^{\dagger} \wedge_{\star} V^{\dagger} \star\left(\psi_{2} \star \overline{\psi_{1}}\right)^{\dagger}\right) \\
& =-i \int_{M} \operatorname{Tr}\left(\psi_{1} \star \overline{\psi_{2}} \gamma_{0} \gamma_{5} \gamma_{0} \star V \wedge_{\star} V \wedge_{\star} V \wedge_{\star} V\right) \\
& =i \int_{M} \operatorname{Tr}\left(\psi_{1} \star \overline{\psi_{2}} \star V \wedge_{\star} V \wedge_{\star} V \wedge_{\star} V \gamma_{5}\right)=\left\langle\psi_{2}, \psi_{1}\right\rangle .
\end{aligned}
$$

In the second equality we have used (graded) cyclicity, the reality condition $V^{\dagger}=\gamma_{0} V \gamma_{0}$, $\left(\psi_{2} \star \overline{\psi_{1}}\right)^{\dagger}=\gamma_{0} \psi_{1} \star \overline{\psi_{2}} \gamma_{0}, \gamma_{0}^{2}=1$ and $\gamma_{5}^{\dagger}=\gamma_{5}$. In the third equality we have used $\gamma_{5} \gamma_{0}=-\gamma_{0} \gamma_{5}$, $\gamma_{0}^{2}=1$ and $\gamma_{5} V=-V \gamma_{5}$.

To show $b$ ) let us set in (2.7) $\lambda=0$ and use that $\left.V\right|_{\lambda=0}=V_{(0)}^{a} \gamma_{a}$ (i.e. that $\tilde{V}^{a}$ vanishes at order $\left.\lambda^{0}\right)$. Using further that the antisymmetrized product of 4 gamma-matrices is $\gamma_{[a} \gamma_{b} \gamma_{c} \gamma_{d]}=$ $-i \gamma_{5} \epsilon_{a b c d}$ and that $\gamma_{5}^{2}=1$ we obtain the desired result.

$c$ ) is a consequence of $b$ ) and the fact that the classical inner product $\int_{M} \overline{\psi_{1}} \psi_{2} \operatorname{vol}_{(0)}$ is nondegenerate.

$\boldsymbol{D}=\mathbf{2}$. The noncommutative twobein and spin connection have the following expansion in terms of the gamma-matrix basis $\left\{1, \gamma_{3}, \gamma_{a}\right\}$ of the 2-dimensional Clifford algebra

$$
V=V^{a} \gamma_{a}, \quad \Omega=\omega \gamma_{3}+\tilde{\omega} 1
$$

We define for $\epsilon=\varepsilon \gamma_{3}+\tilde{\varepsilon} 1$ the $\star$-gauge transformations $\delta_{\epsilon} V:=[\epsilon, V]$ and $\delta_{\epsilon} \Omega:=\mathrm{d} \epsilon+[\epsilon, \Omega]$. As in the case of $D=4$, we had to introduce the extra fields $\tilde{\omega}$ and $\tilde{\varepsilon}$ such that the $\star$-gauge transformations close. Note, however, that we do not need additional terms in the twobein field and thus the interpretation of $V$ as a soldering form remains valid in $D=2$. This will facilitate the study of noncommutative Dirac operators in $D=2$, as compared to $D=4$. We again impose the reality conditions $\epsilon^{\dagger}=-\gamma_{0} \epsilon \gamma_{0}, V^{\dagger}=\gamma_{0} V \gamma_{0}$ and $\Omega^{\dagger}=-\gamma_{0} \Omega \gamma_{0}$.

Definition 2.4. Let $(M, \mathcal{F})$ be a 2-dimensional twisted manifold. A noncommutative Cartan geometry on $(M, \mathcal{F})$ is a pair of Clifford algebra valued one-forms $(V, \Omega)$, satisfying the expansion (2.8), the reality conditions $V^{\dagger}=\gamma_{0} V \gamma_{0}, \Omega^{\dagger}=-\gamma_{0} \Omega \gamma_{0}$ and the limit $\left.\tilde{\omega}\right|_{\lambda=0}=0$.

Let us now consider Dirac fields, which in the case of $D=2$ are functions $\psi \in C^{\infty}\left(M, \mathbb{C}^{2}\right)$ with values in the fundamental representation of the $D=2$ Clifford algebra. The Dirac adjoint is $\bar{\psi}:=\psi^{\dagger} \gamma_{0}$ and $\star$-gauge transformations act on $\psi$ and $\bar{\psi}$ via $\delta_{\epsilon} \psi:=\epsilon \star \psi$ and $\delta_{\epsilon} \bar{\psi}=-\bar{\psi} \star \epsilon$. We define in analogy to (2.7) a $\star$-gauge invariant and hermitian inner product

$$
\left\langle\psi_{1}, \psi_{2}\right\rangle:=\int_{M} \operatorname{Tr}\left(\psi_{2} \star \overline{\psi_{1}} \star V \wedge_{\star} V \gamma_{3}\right) .
$$

For $\lambda=0$ we obtain the usual inner product $\left\langle\psi_{1}, \psi_{2}\right\rangle=\int_{M} \overline{\psi_{1}} \psi_{2} \operatorname{vol}_{(0)}+\mathcal{O}(\lambda)$, since $V \wedge_{\star}$ $\left.V \gamma_{3}\right|_{\lambda=0}=V_{(0)} \wedge V_{(0)} \gamma_{3}=V_{(0)}^{a} \wedge V_{(0)}^{b} \epsilon_{a b} \gamma_{3}^{2}=\operatorname{vol}_{(0)}$. 


\section{Noncommutative Dirac operators}

As a first step, we will give three explicit candidate definitions for noncommutative Dirac operators in $D=2$ and $D=4$. These are obtained by using techniques of noncommutative differential geometry and twist deformation quantization. Depending on taste and point of view, either of them may be seen as a valid extension of the classical Dirac operator to the noncommutative setting. This shows that a more abstract characterization of noncommutative Dirac operators is needed, and we develop in the second step what we believe is a minimal set of axioms for such operators. This will already rule out the classical Dirac operator along with one of our candidates, and we show that the remaining two indeed meet our criteria for noncommutative Dirac operators.

\subsection{Explicit candidates}

The following set of candidates for noncommutative Dirac operators should show, how focusing on different aspects of noncommutative differential geometry and twist deformation quantization leads to different constructions. It is not meant to be exhaustive.

The Aschieri-Castellani Dirac operator. The first operator we consider is motivated by the noncommutative $D=4$ Dirac field action proposed in [1], which reads

$$
S^{\mathrm{AC}}=-4 \int_{M} \operatorname{Tr}\left(\left(\mathrm{d}_{\Omega} \psi\right) \star \bar{\psi} \wedge_{\star} V \wedge_{\star} V \wedge_{\star} V \gamma_{5}\right),
$$

where $\mathrm{d}_{\Omega} \psi:=\mathrm{d} \psi-\Omega \star \psi$ is the $\star$-covariant differential acting on Dirac fields. Since the inner product $(2.7)$ is non-degenerate, we can define a differential operator $\not^{\mathrm{AC}}: C^{\infty}\left(M, \mathbb{C}^{4}\right) \rightarrow$ $C^{\infty}\left(M, \mathbb{C}^{4}\right)$ by requiring that, for all $\psi_{1}$ of compact support,

$$
\left\langle\psi_{1}, \not D^{\mathrm{AC}} \psi_{2}\right\rangle=-4 \int_{M} \operatorname{Tr}\left(\left(\mathrm{d}_{\Omega} \psi_{2}\right) \star \overline{\psi_{1}} \wedge_{\star} V \wedge_{\star} V \wedge_{\star} V \gamma_{5}\right)
$$

This yields exactly the equation of motion operator which is obtained by varying the action (3.1) with respect to $\bar{\psi}$. The construction in $D=2$ is fully analogous. The action then reads

$$
S^{\mathrm{AC}}=2 i \int_{M} \operatorname{Tr}\left(\left(\mathrm{d}_{\Omega} \psi\right) \star \bar{\psi} \wedge_{\star} V \gamma_{3}\right),
$$

and since the inner product (2.9) is also non-degenerate, we can define a differential operator $\not D^{\mathrm{AC}}: C^{\infty}\left(M, \mathbb{C}^{2}\right) \rightarrow C^{\infty}\left(M, \mathbb{C}^{2}\right)$ by requiring that, for all $\psi_{1}$ of compact support,

$$
\left\langle\psi_{1}, \not D^{\mathrm{AC}} \psi_{2}\right\rangle=2 i \int_{M} \operatorname{Tr}\left(\left(\mathrm{d}_{\Omega} \psi_{2}\right) \star \overline{\psi_{1}} \wedge_{\star} V \gamma_{3}\right) \text {. }
$$

The contraction Dirac operator. For our next operator we shall follow closely the usual construction of a Dirac operator on commutative spacetimes, which goes as follows: Let $V_{(0)}=$ $V_{(0)}^{a} \gamma_{a}$ be a classical vielbein, $\Omega_{(0)}$ a classical spin connection and let us denote by $V_{(0)}^{-1}=E_{(0) a} \gamma^{a}$ the inverse vielbein. The classical Dirac operator is $\not D_{(0)} \psi=i \gamma^{a} \nabla_{(0) a} \psi=i \gamma^{a}\left(E_{(0) a}(\psi)-\Omega_{(0) a} \psi\right)$, where we have expressed $\Omega_{(0)}$ in the vielbein basis $\Omega_{(0)}=V^{a} \Omega_{(0) a}$. Notice that this operator can be written in an index-free form $\nsupseteq_{(0)} \psi=i \iota_{V_{(0)}^{-1}}\left(\mathrm{~d} \psi-\Omega_{(0)} \psi\right)$, where $\iota$ is the classical contraction operator (interior product).

Using the deformed contraction operator $\iota^{\star}$ between vector fields and one-forms as defined in (2.4), we generalize the above construction to the noncommutative setting. For this we define the $\star$-inverse vielbein $E_{a}$ by the $\star$-contraction condition $\iota_{E_{a}}^{\star}\left(V^{b}\right)=\delta_{a}^{b}$. We collect all $E_{a}$ in 
the Clifford algebra valued vector field $V^{-1_{\star}}:=E_{a} \gamma^{a}$. Following the same strategy as in the classical case, we define a differential operator $\not^{\text {contr }}$ by

$$
\not \not^{\text {contr }} \psi:=i \iota_{V}^{\star} 1_{\star}\left(\mathrm{d}_{\Omega} \psi\right)=i \gamma^{a} \iota_{E_{a}}^{\star}\left(\mathrm{d}_{\Omega} \psi\right) .
$$

The construction outlined above is valid as it stands in $D=2$. For $D=4$ the following remark is in order: As seen in (2.5), the noncommutative vierbein field has an extra field $\widetilde{V}^{a}$. This implies that $V$ is locally a $4 \times 8$-matrix and hence there is no unique $\star$-inverse $V^{-1_{\star}}$. Since invertibility of the vielbein is essential in classical vielbein gravity, this may be seen as a shortcoming of the noncommutative $D=4$ vielbein gravity formulated in [1]. There have been attempts to overcome this issue by using Seiberg-Witten maps [3], which, however, obscure the noncommutative differential geometry and in practice require an expansion in the deformation parameter to some fixed order, so they are not convenient for our purpose. We shall instead take the following approach: We restrict the class of allowed noncommutative Cartan geometries $(V, \Omega)$ to those satisfying $V=V^{a} \gamma_{a}$. We are aware that this restriction is not invariant under *-gauge transformations (in $D=4$ ) and hence it is not convenient for dynamical noncommutative $D=4$ vielbein gravity. However, for a given fixed noncommutative Cartan geometry $(V, \Omega)$ it certainly makes sense to assume a $V$ of this special form.

The deformed Dirac operator. The last noncommutative Dirac operator is motivated by the framework of Connes for noncommutative spin geometry [13], where the Dirac operator enters as a fundamental degree of freedom of the theory. It is obtained by deforming the classical Dirac operator $\nsupseteq_{(0)}$ via the techniques developed in [6]. More precisely, denoting the inverse twist by $\mathcal{F}^{-1}=\bar{f}^{\alpha} \otimes \bar{f}_{\alpha}$, we define the deformed Dirac operator by applying the deformation map constructed in [6]

$$
\not D^{\mathcal{F}} \psi:=\left(\bar{f}^{\alpha} \triangleright D_{(0)}\right) \bar{f}_{\alpha}(\psi)=\not D_{(0)} \psi+\frac{i \lambda}{2} \Theta^{\alpha \beta}\left(X_{\alpha} \triangleright \not_{(0)}\right) X_{\beta}(\psi)+\cdots,
$$

where $X_{\alpha} \triangleright \not_{(0)}:=X_{\alpha} \circ \nsupseteq_{(0)}-\not_{(0)} \circ X_{\alpha}$ is the adjoint action.

\subsection{Abstract consideration}

The variety of different generalizations of the classical Dirac operator to the noncommutative setting provided above clearly calls for a more precise definition of what we actually mean by a noncommutative Dirac operator. As a reasonable starting point, we are looking for linear differential operators $\not D: C^{\infty}\left(M, \mathbb{C}^{N}\right) \rightarrow C^{\infty}\left(M, \mathbb{C}^{N}\right)$ acting on Dirac fields $\psi \in C^{\infty}\left(M, \mathbb{C}^{N}\right)$ ( $N$ is the dimension of the fundamental representation of the Clifford algebra), subject to certain conditions generalizing the properties of the classical Dirac operator. Thus, we naturally start by generalizing some relevant notions to the noncommutative setting, beginning with the notion of a first-order differential operator.

Definition 3.1. Let $(M, \mathcal{F})$ be a twisted manifold. A differential operator $\not D: C^{\infty}\left(M, \mathbb{C}^{N}\right) \rightarrow$ $C^{\infty}\left(M, \mathbb{C}^{N}\right)$ is called a first-order noncommutative differential operator, if for all $\psi \in C^{\infty}\left(M, \mathbb{C}^{N}\right)$ and $a \in C^{\infty}(M)$,

$$
\not D(\psi \star a)=\not D(\psi) \star a+\iota_{Q_{\psi}}^{\star}(\mathrm{d} a),
$$

where $Q_{\psi}$ is a spinor-valued vector field on $M$ and the $\star$-contraction is defined in (2.4).

For $\lambda=0$ we obtain from (3.5) the usual Leibniz rule property of a first-order differential operator. Written in local coordinates it reads $\not D(\psi a)=\not D(\psi) a+Q_{\psi}^{\mu} \partial_{\mu} a$. Hence, (3.5) promotes this property to the realm of twisted manifolds. Note that a first-order noncommutative differential operator is not necessarily a first-order differential operator in the usual sense (cf. the examples 
in the sections below). It can and in general must contain higher order derivatives, but these are restricted by the form of the twist $\mathcal{F}$.

The second notion we want to generalize aims to capture more of the essence of the classical Dirac operator. Namely, that it is constructed from purely geometric data in a natural way. We will formalize the requirement that a noncommutative Dirac operator should be constructed only from the data of the noncommutative Cartan geometry $(V, \Omega)$ and the twisted manifold $(M, \mathcal{F})$ in a geometric (natural) way as follows ${ }^{2}$ :

Definition 3.2. Let $(V, \Omega)$ be a noncommutative Cartan geometry on a twisted manifold $(M, \mathcal{F})$. A differential operator $\not D: C^{\infty}\left(M, \mathbb{C}^{N}\right) \rightarrow C^{\infty}\left(M, \mathbb{C}^{N}\right)$ is called a geometric noncommutative differential operator if it is constructed from the noncommutative vielbein $V$ and the $\star$-covariant differential $\mathrm{d}_{\Omega}$ in terms of the operations of twisted noncommutative geometry.

We can now state our general definition for noncommutative Dirac operators, which combines the properties introduced above with the natural demand that the standard Dirac operator should be recovered in the commutative limit:

Definition 3.3. A noncommutative Dirac operator on a noncommutative Cartan geometry $(V, \Omega)$ over a twisted manifold $(M, \mathcal{F})$ is a differential operator $\not D: C^{\infty}\left(M, \mathbb{C}^{N}\right) \rightarrow C^{\infty}\left(M, \mathbb{C}^{N}\right)$, such that

1) $\not D$ is a first-order noncommutative differential operator,

2) $\not D$ is a geometric noncommutative differential operator,

3) $\not D$ reproduces the classical Dirac operator $\not_{(0)}$ corresponding to $\left(V_{(0)}, \Omega_{(0)}\right)$ for $\lambda=0$.

Having stated this definition, the first two questions one could ask are, firstly, whether there are noncommutative Dirac operators in this sense at all, and, secondly, whether the requirements are possibly trivial altogether. In the remaining part of this subsection we will answer these two questions, focusing on $D=2$ and $D=4$.

The first question is easily answered by providing an explicit construction which satisfies the demands of Definition 3.3:

Proposition 3.4. Let $(M, \mathcal{F})$ be any 2- or 4-dimensional twisted manifold and $(V, \Omega)$ any noncommutative Cartan geometry. Then the operator $\not D^{\mathrm{AC}}$ defined in (3.3) and (3.2) is a noncommutative Dirac operator according to Definition 3.3.

Proof. We give the proof for $D=2$, and note that it follows analogously for $D=4$. We have to check the three conditions in Definition 3.3. For property 1) let us evaluate the following inner product

$$
\begin{aligned}
\left\langle\psi_{1}, \not D^{\mathrm{AC}}\left(\psi_{2} \star a\right)\right\rangle & =2 i \int_{M} \operatorname{Tr}\left(\left(\mathrm{d}_{\Omega}\left(\psi_{2} \star a\right)\right) \star \overline{\psi_{1}} \wedge \star V \gamma_{3}\right) \\
& =2 i \int_{M} \operatorname{Tr}\left(\left(\left(\mathrm{d}_{\Omega} \psi_{2}\right) \star a+\psi_{2} \star \mathrm{d} a\right) \star \overline{\psi_{1}} \wedge_{\star} V \gamma_{3}\right) \\
& =\left\langle\psi_{1}, \not D^{\mathrm{AC}}\left(\psi_{2}\right) \star a\right\rangle+2 i \int_{M} \operatorname{Tr}\left(\psi_{2} \star \mathrm{d} a \star \overline{\psi_{1}} \wedge_{\star} V \gamma_{3}\right) .
\end{aligned}
$$

The proof would follow if we could show that the condition

$$
V \wedge_{\star} V \gamma_{3} \star \iota_{Q_{\psi_{2}}}^{\star}(\omega)=-2 i V \gamma_{3} \star \psi_{2} \wedge_{\star} \omega, \quad \text { for all } \omega \in \Omega^{1}(M),
$$

\footnotetext{
${ }^{2}$ This can be made more precise in a category theoretical framework for noncommutative Cartan geometries on twisted manifolds, where a natural differential operator could be defined in terms of a natural transformation between the section functors of the Dirac bundles.
} 
defines a unique spinor-valued vector field $Q_{\psi_{2}}$ on $M$. This is indeed the case by the following argument: Notice that $V \wedge_{\star} V \gamma_{3}$ is equal to the classical volume form vol $(0)$ at order $\lambda^{0}$. Since this form is non-degenerate, the condition (3.6) determines a unique spinor-valued function $\iota_{Q_{\psi_{2}}}^{\star}(\omega)$, for any $\omega \in \Omega^{1}(M)$. The operation $\iota_{Q_{\psi_{2}}}^{\star}$ is right linear under the $\star$-multiplication by $C^{\infty}(M)$, for all $\omega \in \Omega^{1}(M)$ and $a \in C^{\infty}(M)$,

$$
V \wedge_{\star} V \gamma_{3} \star \iota_{Q_{\psi_{2}}}^{\star}(\omega \star a)=-2 i V \gamma_{3} \star \psi_{2} \wedge_{\star} \omega \star a=V \wedge_{\star} V \gamma_{3} \star \iota_{Q_{\psi_{2}}}^{\star}(\omega) \star a .
$$

Since the space of vector fields is exactly the dual module of the module of one-forms, $Q_{\psi_{2}}$ is a spinor-valued vector field.

Property 2) is clear: We have used only $\star$-products, $\wedge_{\star}$-products, integrals $\int_{M}$, vielbeins $V$ and $\star$-covariant differentials $\mathrm{d}_{\Omega}$ in order to define $\not^{\mathrm{AC}}$.

To prove property 3) we consider (3.3) at $\lambda=0$. We expand $\left.\mathrm{d}_{\Omega} \psi_{2}\right|_{\lambda=0}$ in the twobein basis $V_{(0)}^{a}$, i.e. $\left.\mathrm{d}_{\Omega} \psi_{2}\right|_{\lambda=0}=V_{(0)}^{a}\left(E_{(0) a}\left(\psi_{2}\right)-\Omega_{(0) a} \psi_{2}\right)$, where $E_{(0) a}$ is the inverse of $V_{(0)}^{a}$, which is a vector field. We obtain

$$
\begin{aligned}
\left.\left\langle\psi_{1}, \not D^{\mathrm{AC}} \psi_{2}\right\rangle\right|_{\lambda=0} & =2 i \int_{M} \operatorname{Tr}\left(V_{(0)}^{b}\left(E_{(0) b}\left(\psi_{2}\right)-\Omega_{(0) b} \psi_{2}\right) \overline{\psi_{1}} \wedge V_{(0)}^{a} \gamma_{a} \gamma_{3}\right) \\
& =2 i \int_{M} \overline{\psi_{1}} \epsilon_{a c} \gamma^{c}\left(E_{(0) b}\left(\psi_{2}\right)-\Omega_{(0) b} \psi_{2}\right) V_{(0)}^{a} \wedge V_{(0)}^{b} \\
& =\int_{M} \overline{\psi_{1}} i \gamma^{a}\left(E_{(0) a}\left(\psi_{2}\right)-\Omega_{(0) a} \psi_{2}\right) \operatorname{vol}_{(0)}=\int_{M} \overline{\psi_{1}}\left(\not D_{(0)} \psi_{2}\right) \operatorname{vol}_{(0)} .
\end{aligned}
$$

Bearing in mind the remark on the $D=4$ case below Equation (3.4), also the contraction Dirac operator is valid in the sense of Definition 3.3:

Proposition 3.5. Let $(M, \mathcal{F})$ be a twisted manifold of dimension 2 or 4 , and $(V, \Omega)$ a noncommutative Cartan geometry, such that $V=V^{a} \gamma_{a}$. Then the operator $\not^{\text {contr }}$ defined in (3.4) is a noncommutative Dirac operator according to Definition 3.3.

Proof. We have to check the three conditions in Definition 3.3. Property 1) follows from a short calculation

$$
\not D^{\text {contr }}(\psi \star a)=i \iota_{V^{-1} \star}^{\star}\left(\left(\mathrm{d}_{\Omega} \psi\right) \star a+\psi \star \mathrm{d} a\right)=\not D^{\operatorname{contr}}(\psi) \star a+\iota_{Q_{\psi}}^{\star}(\mathrm{d} a),
$$

where $Q_{\psi}=i V^{-1_{\star}} \star \psi$.

Property 2) is clear: We have only used $V, \mathrm{~d}_{\Omega}$, the $\star$-inverse $V^{-1_{\star}}$ (defined via $\iota_{\star}$ ) and $\iota_{\star}$ to construct $\not^{\text {contr }}$. Furthermore, property 3 ) is a consequence of the fact that all operations entering (3.4) reduce for $\lambda=0$ to the corresponding classical operations.

To answer the second question and show that the requirements are indeed not vacuous, we show that, quite expectedly, the classical Dirac operator $\not_{(0)}$ fails to meet our criteria. It will nevertheless be instructive to see which conditions are violated. Furthermore, we will see that the deformed Dirac operator $\not^{\mathcal{F}}$ is ruled out as well. Let us first note that property 3), controlling the classical limit, is satisfied by both of these operators. In order to understand if the classical Dirac operator satisfies property 1), we expand up to first order in the deformation parameter $\lambda$, which yields

$$
\begin{aligned}
\not D_{(0)}(\psi \star a)= & \not D_{(0)}\left(\psi a+\frac{i \lambda}{2} \Theta^{\alpha \beta} X_{\alpha}(\psi) X_{\beta}(a)\right)+\mathcal{O}\left(\lambda^{2}\right) \\
= & \not D_{(0)}(\psi) a+\frac{i \lambda}{2} \Theta^{\alpha \beta} \not D_{(0)}\left(X_{\alpha}(\psi)\right) X_{\beta}(a) \\
& +\iota_{V_{(0)}^{-1} \psi}(\mathrm{d} a)+\frac{i \lambda}{2} \Theta^{\alpha \beta} \iota_{V_{(0)}^{-1} X_{\alpha}(\psi)}\left(X_{\beta}(\mathrm{d} a)\right)+\mathcal{O}\left(\lambda^{2}\right) .
\end{aligned}
$$


For $\not_{(0)}$ to be a first-order noncommutative differential operator the $X_{\alpha}$ have to commute with $\not_{(0)}$ and $V_{(0)}$, i.e. the twist has to be generated completely by Killing vector fields. This shows that for generic noncommutative Cartan geometries $(V, \Omega)$ on twisted manifolds $(M, \mathcal{F})$ the classical Dirac operator $\not_{(0)}$ is not a first-order noncommutative differential operator and in particular not a noncommutative Dirac operator.

The deformed Dirac operator, on the other hand, is a first-order noncommutative differential operator, since

$$
\not D^{\mathcal{F}}(\psi \star a)=\not D^{\mathcal{F}}(\psi) \star a+\iota_{i V_{(0)}^{-1} \star \psi}(\mathrm{d} a) .
$$

However, like the classical Dirac operator it fails to satisfy property 2), as in the construction of $\not D_{(0)}$ and $\not D^{\mathcal{F}}$ undeformed covariant differentials and contraction operators appear. Summing up, our axioms above are satisfied by $\not D^{\mathrm{AC}}$ and $\not^{\text {contr }}$, but the deformed and classical Dirac operator fails in the general to be a noncommutative Dirac operator. We will turn to the question for the remaining freedom to choose a Dirac operator in the next sections.

\section{Comparing the noncommutative Dirac operators}

\subsection{Non-uniqueness in the general case}

Having established two examples of noncommutative Dirac operators on noncommutative Cartan geometries $(V, \Omega)$ over twisted manifolds $(M, \mathcal{F})$, we shall now show that they do not coincide in general. Our strategy is to calculate explicitly the two noncommutative Dirac operators $\not^{\mathrm{AC}}$ and $\not D^{\text {contr }}$ for a simple example of $(V, \Omega)$ and $(M, \mathcal{F})$, from which the desired result can be directly read off.

We will start with the 2-dimensional case and consider the noncommutative spacetime known as 'quantum plane'. Let $M=\mathbb{R}^{2}$ and consider the twist $\mathcal{F}$ in (2.1), constructed from $X_{1}=t \partial_{t}$ and $X_{2}=x \partial_{x}$, where $t$ and $x$ are global coordinates. Notice that this twisted manifold $(M, \mathcal{F})$ leads to the commutation relations of the quantum plane, i.e. $t \star x=e^{i \lambda} x \star t$. We equip this twisted manifold with the following noncommutative Cartan geometry: $V=V^{a} \gamma_{a}=\gamma_{0} \mathrm{~d} t+\gamma_{1} \mathrm{~d} x$ and $\Omega=0$ is the unique $\star$-torsion free connection. The $\star$-inverse $E_{a}$ of $V^{a}$ is defined by $\iota_{E_{a}}^{\star}\left(V^{b}\right)=\delta_{a}^{b}$ and it is given by $E_{0}=\partial_{t}, E_{1}=\partial_{x}$. We further find for the $\star$-covariant differential

$\mathrm{d}_{\Omega} \psi=\mathrm{d} \psi=\mathrm{d} t \star e^{-\frac{i \lambda}{2} x \partial_{x}} \partial_{t} \psi+\mathrm{d} x \star e^{\frac{i \lambda}{2} t \partial_{t}} \partial_{x} \psi$. This leads to the following contraction Dirac operator (3.4)

$$
\not D^{\text {contr }} \psi=i\left(\gamma^{0} e^{-\frac{i \lambda}{2} x \partial_{x}} \partial_{t} \psi+\gamma^{1} e^{\frac{i \lambda}{2} t \partial_{t}} \partial_{x} \psi\right) .
$$

In order to compare our two noncommutative Dirac operators, we also evaluate the AschieriCastellani Dirac operator (3.3) for this model. Using that $\mathrm{d} t \wedge_{\star} \mathrm{d} x=e^{\frac{i \lambda}{2}} \mathrm{~d} t \wedge \mathrm{d} x=e^{\frac{i \lambda}{2}} \mathrm{vol} / 2$, with vol $=\epsilon_{a b} V^{a} \wedge V^{b}$ denoting the volume form, we obtain for the inner product (2.9)

$$
\left\langle\psi_{1}, \psi_{2}\right\rangle=\cos (\lambda / 2) \int_{M} \overline{\psi_{1}} \star \operatorname{vol} \star \psi_{2} .
$$

Furthermore, evaluating (3.3) we obtain

$$
\left\langle\psi_{1}, \not D^{\mathrm{AC}} \psi_{2}\right\rangle=i \int_{M} \overline{\psi_{1}} \star \operatorname{vol} \star\left(e^{-\frac{i \lambda}{2}} \gamma^{0} e^{-\frac{i \lambda}{2} x \partial_{x}} \partial_{t} \psi_{2}+e^{\frac{i \lambda}{2}} \gamma^{1} e^{\frac{i \lambda}{2} t \partial_{t}} \partial_{x} \psi_{2}\right),
$$

which yields the Aschieri-Castellani Dirac operator on the quantum plane

$$
\not D^{\mathrm{AC}} \psi=\frac{i}{\cos (\lambda / 2)}\left(e^{-\frac{i \lambda}{2}} \gamma^{0} e^{-\frac{i \lambda}{2} x \partial_{x}} \partial_{t} \psi+e^{\frac{i \lambda}{2}} \gamma^{1} e^{\frac{i \lambda}{2} t \partial_{t}} \partial_{x} \psi\right) \text {. }
$$


Comparing (4.1) and (4.3) we observe that the noncommutative Dirac operators $\not D^{\text {contr }}$ and $\not D^{\mathrm{AC}}$ do not coincide. Notice that the difference is not just in the overall factor, but the two terms have also acquired different phases. With the 4-dimensional analog of the quantum plane and a similar calculation, we obtain the following result.

Proposition 4.1. The two noncommutative Dirac operators $\not^{\mathrm{AC}}$ and $\not^{\text {contr }}$ do not coincide for generic noncommutative Cartan geometries $(V, \Omega)$ over twisted manifolds $(M, \mathcal{F})$ of dimension 2 or 4 .

\subsection{The formal self-adjointness condition}

In regard of the non-uniqueness result above we try to include stronger conditions on noncommutative Dirac operators in order to single out a preferred choice. We shall focus in this subsection only on one strongly motivated extra condition, which is formal self-adjointness. This condition is essential for associating to a noncommutative Dirac operator a quantum field theory of noncommutative Dirac fields, see Section 5. Notice that also in a Riemannian setting, a formal self-adjointness condition is an important ingredient for understanding the spectral theory of Dirac operators.

Definition 4.2. Let $(M, \mathcal{F})$ be a twisted manifold and $(V, \Omega)$ a noncommutative Cartan geometry on $(M, \mathcal{F})$. A noncommutative Dirac operator $\not D$ is called formally self-adjoint, if it satisfies $\left\langle\psi_{1}, \not D \psi_{2}\right\rangle=\left\langle\not D \psi_{1}, \psi_{2}\right\rangle$, for all Dirac fields $\psi_{1}, \psi_{2}$ with compactly overlapping support. Here $\langle\cdot, \cdot\rangle$ is the inner product defined for $D=2$ in (2.9) and for $D=4$ in (2.7).

Notice that the classical Dirac operator $\not_{(0)}$ is formally self-adjoint with respect to the classical inner product $\left.\langle\cdot, \cdot\rangle\right|_{\lambda=0}$ if the spin connection is torsion free. For the Aschieri-Castellani Dirac operator we obtain the analogous property in the twisted setting.

Proposition 4.3. Let $(M, \mathcal{F})$ be any twisted manifold of dimension 2 or 4 , and let $(V, \Omega)$ be a noncommutative Cartan geometry that is $\star$-torsion free, i.e. $\mathrm{d}_{\Omega} V=0$. Then the noncommutative Dirac operator defined in (3.3) and (3.2) is formally self-adjoint.

Proof. We show this statement by the following calculation for the 2-dimensional case

$$
\begin{aligned}
\left\langle\not D^{\mathrm{AC}} \psi_{1}, \psi_{2}\right\rangle & =\left\langle\psi_{2}, \not D^{\mathrm{AC}} \psi_{1}\right\rangle^{*}=2 i \int_{M} \operatorname{Tr}\left(\gamma_{3}^{\dagger} V^{\dagger} \star \gamma_{0} \psi_{2} \wedge_{\star} \mathrm{d}_{\Omega} \overline{\psi_{1}} \gamma_{0}\right) \\
& =2 i \int_{M} \operatorname{Tr}\left(\gamma_{3} \gamma_{0} V \star \psi_{2} \wedge_{\star} \mathrm{d}_{\Omega} \overline{\psi_{1}} \gamma_{0}\right)=-2 i \int_{M} \operatorname{Tr}\left(\psi_{2} \star \mathrm{d}_{\Omega} \overline{\psi_{1}} \wedge_{\star} V \gamma_{3}\right) \\
& =-2 i \int_{M} \mathrm{~d} \operatorname{Tr}\left(\psi_{2} \star \overline{\psi_{1}} \wedge_{\star} V \gamma_{3}\right)+2 i \int_{M} \operatorname{Tr}\left(\mathrm{d}_{\Omega} \psi_{2} \star \overline{\psi_{2}} \wedge_{\star} V \gamma_{3}\right) \\
& =\left\langle\psi_{1}, \not D^{\mathrm{AC}} \psi_{2}\right\rangle .
\end{aligned}
$$

In the first equality we have used hermiticity of the inner product. In the second equality we have used that $*$ is a graded involution on the deformed differential forms $\left(\Omega^{\bullet}(M), \wedge_{\star}, \mathrm{d}\right)$ as well as $\left(\mathrm{d}_{\Omega} \psi_{1} \star \overline{\psi_{2}}\right)^{\dagger}=\gamma_{0} \psi_{2} \star \mathrm{d}_{\Omega} \overline{\psi_{1}} \gamma_{0}$, which follows from the hermiticity condition $\Omega^{\dagger}=-\gamma_{0} \Omega \gamma_{0}$. Then for the third step we used $V^{\dagger}=\gamma_{0} V \gamma_{0}, \gamma_{3}^{\dagger}=\gamma_{3}$ and $\gamma_{0}^{2}=1$, and in the fourth one graded cyclicity (2.3) and twice $\gamma_{3} \gamma_{a}=-\gamma_{a} \gamma_{3}$. In the fifth equality we made use of the graded Leibniz rule of $\mathrm{d}_{\Omega}$ and the $\star$-torsion constraint $\mathrm{d}_{\Omega} V=0$. The last step is simply Stokes' theorem. The proof for the 4-dimensional case is once again fully analogous.

We will now show that our second example of a noncommutative Dirac operator, that is the contraction Dirac operator, does not satisfy the formal self-adjointness condition on generic $\star$-torsion free noncommutative Cartan geometries $(V, \Omega)$. To this end we again consider the 
quantum plane as a simple example. The contraction Dirac operator is given in (4.1) and using the explicit form of the inner product (4.2) we obtain for the formal adjoint of the contraction Dirac operator

$$
\left(\not D^{\mathrm{contr}}\right)^{*} \psi=i\left(e^{-i \lambda} \gamma^{0} e^{-\frac{i \lambda}{2} x \partial_{x}} \partial_{t} \psi+e^{i \lambda} \gamma^{1} e^{\frac{i \lambda}{2} t \partial_{t}} \partial_{x} \psi\right)
$$

The differential operators $\left(\not D^{\text {contr }}\right)^{*}$ and $\not \not^{\text {contr }}$ (cf. (4.1)) do not agree, hence the contraction Dirac operator is not formally self-adjoint on the quantum plane. With the analogous investigation of the 4-dimensional analog of the quantum plane, this leads to the following conclusion.

Proposition 4.4. The noncommutative Dirac operator $\not^{\text {contr }}$ is not formally self-adjoint for generic $\star$-torsion free noncommutative Cartan geometries $(V, \Omega)$ over twisted manifolds $(M, \mathcal{F})$ of dimension 2 or 4 . Hence, the Aschieri-Castellani Dirac operator is the preferred choice among our two noncommutative Dirac operators.

\subsection{Semi-Killing deformations as a special case}

As noted in $[2,26,33]$, any metric field solving the classical Einstein equations also solves the noncommutative Einstein equations [4] if the twist is semi-Killing. Explicitly, an Abelian twist (2.1) is semi-Killing if $\Theta^{\alpha \beta} X_{\alpha} \otimes X_{\beta} \in \Xi \otimes \mathfrak{K}+\mathfrak{K} \otimes \Xi$, where $\Xi$ is the Lie algebra of vector fields on $M$ and $\mathfrak{K}:=\left\{X \in \Xi: \mathcal{L}_{X}(V)=0\right.$ and $\left.\mathcal{L}_{X}(\Omega)=0\right\}$ is the Killing Lie algebra. Using the canonical form of $\Theta^{\alpha \beta}(2.2)$, this condition is equivalent to requiring that either $X_{2 n}$ or $X_{2 n-1}$ is a Killing vector field, for all $n=1,2, \ldots$.

Let now $(V, \Omega)$ be a noncommutative Cartan geometry over a twisted manifold $(M, \mathcal{F})$, such that $\mathcal{F}$ is semi-Killing. We notice that in this case $\left(V=V^{a} \gamma_{a}, \Omega=\frac{1}{4} \omega^{a b} \gamma_{a b}\right)$ solves the noncommutative Einstein equations (in vielbein form) [1] whenever it solves the commutative Einstein equations. In the following we study our noncommutative Dirac operators for this particular class of examples and show that they coincide. Let us denote by $E_{a}$ the basis for the vector fields on $M$ which is specified by the undeformed contraction condition $\iota_{E_{a}}\left(V^{b}\right)=\delta_{a}^{b}$. This condition implies that $\mathcal{L}_{X}\left(E_{a}\right)=0$ for all $X \in \mathfrak{K}$ and, hence, also the deformed contraction condition $\iota_{E_{a}}^{\star}\left(V^{b}\right)=\delta_{a}^{b}$ holds true for semi-Killing twists. The $\star$-inverse vierbein therefore reads $V^{-1_{\star}}=E_{a} \gamma^{a}$. We define the components $\Omega_{a}$ of the spin connection by $\Omega=: V^{a} \Omega_{a}$. The conditions $\mathcal{L}_{X}(\Omega)=0$ and $\mathcal{L}_{X}(V)=0$ imply that $\mathcal{L}_{X}\left(\Omega_{a}\right)=0$, for all $X \in \mathfrak{K}$, and thus $\Omega=V^{a} \Omega_{a}=V^{a} \star \Omega_{a}$. Furthermore, we define the differential operator $E_{a}^{\star}$ by $E_{a}^{\star}(\psi):=\iota_{E_{a}}^{\star}(\mathrm{d} \psi)$ and we obtain that $\iota_{E_{a}}^{\star}\left(\mathrm{d}_{\Omega} \psi\right)=E_{a}^{\star}(\psi)-\Omega_{a} \star \psi$. The contraction Dirac operator (3.4) expressed in this basis reads

$$
\not D^{\text {contr }} \psi=i \gamma^{a} \iota_{E_{a}}^{\star}\left(\mathrm{d}_{\Omega} \psi\right)=i \gamma^{a}\left(E_{a}^{\star}(\psi)-\Omega_{a} \star \psi\right) .
$$

Let us also compute explicitly the Aschieri-Castellani Dirac operator (3.3) for this class of examples. We show the calculation for the 2-dimensional case and note that it is fully analogous for $D=4$. Due to the semi-Killing property we have $V \wedge_{\star} V \gamma_{3}=V \wedge V \gamma_{3}=$ vol, with $\mathrm{vol}=\epsilon_{a b} V^{a} \wedge V^{b}$. Hence, the inner product (2.9) reads

$$
\left\langle\psi_{1}, \psi_{2}\right\rangle=\int_{M} \overline{\psi_{1}} \star \mathrm{vol} \star \psi_{2} .
$$

Using $V \gamma_{3}=-V^{a} \epsilon_{a b} \gamma^{b}$ we find that (3.3) simplifies to

$$
\left\langle\psi_{1}, \not D^{\mathrm{AC}} \psi_{2}\right\rangle=2 i \int_{M} \overline{\psi_{1}} \star V^{a} \epsilon_{a b} \gamma^{b} \wedge_{\star} \mathrm{d}_{\Omega} \psi_{2}=\int_{M} \overline{\psi_{1}} \star \operatorname{vol} \star i \gamma^{a} \iota_{E_{a}}\left(\mathrm{~d}_{\Omega} \psi_{2}\right) .
$$


This and (4.5) implies that $\not D^{\mathrm{AC}}$ coincides with $\not^{\text {contr }}$ (cf. (4.4)) for semi-Killing twists. Notably, also the deformed Dirac operator $\not^{\mathcal{F}}$ (obtained by deforming the classical Dirac operator $\not_{(0)}$ corresponding to $(V, \Omega)$ ) coincides with $\not^{\mathrm{AC}}$ and $\not^{\text {contr }}$, even though it in general fails to satisfy our requirements. We would like to stress that for generic semi-Killing twists the noncommutative Dirac operators do not coincide with the classical one $\not_{(0)}$ corresponding to $(V, \Omega)$, cf. Section 6 for explicit examples. In summary, we have obtained the following

Proposition 4.5. For semi-Killing twists the two noncommutative Dirac operators $\not^{\mathrm{AC}}$ and $\not D^{\text {contr }}$ coincide. Even more, in this case these operators also coincide with the operator $\not^{\mathcal{F}}$, which in general does not satisfying our axioms. For practical purposes one can therefore choose the technically most convenient one.

If, moreover, all vector fields $X_{\alpha}$ in the twist $\mathcal{F}$ are Killing, then the differential operator $E_{a}^{\star}$ defined by $E_{a}^{\star}(\psi)=\iota_{E_{a}}^{\star}(\mathrm{d} \psi)$ coincides with the vector field $E_{a}$ (the inverse vierbein). Since also $\Omega_{a} \star \psi=\Omega_{a} \psi$, we obtain for the contraction Dirac operator (4.4) $\not D^{\text {contr }} \psi=i \gamma^{a}\left(E_{a}(\psi)-\Omega_{a} \psi\right)$. Hence, the operator $\not D^{\text {contr }}$ coincides in the Killing case with the classical Dirac operator $\not_{(0)}$ corresponding to $(V, \Omega)$. Since actual Killing twists are contained in the class of semi-Killing twists, we have further $\not^{\text {contr }}=\not D^{\mathrm{AC}}=\not^{\mathcal{F}}=\not_{(0)}$ for Killing twists.

Applying this result to the noncommutative (Riemannian) space considered in [14], which in our notation corresponds to a Killing twist deformation of the sphere, we also come to the conclusion that the classical Dirac operator is a suitable noncommutative Dirac operator for this model. Moreover, all of our deformed constructions for noncommutative Dirac operators reduce in this case to the classical one, hence there is (within our class of operators) no alternative choice. The main reason behind this is, of course, the invariance of the Cartan geometry and the classical Dirac operator under the (Killing) vector fields entering the twist.

\section{Quantum field theory of noncommutative Dirac fields}

In [27] we have constructed retarded/advanced Green's operators as well as the solution space of deformed wave equations, which led to the construction of noncommutative Klein-Gordon quantum field theories. In this section we generalize these results to noncommutative Dirac operators, and hence to noncommutative quantum field theories of Dirac fields.

We start with a general linear differential operator $P: C^{\infty}\left(M, \mathbb{C}^{N}\right) \rightarrow C^{\infty}\left(M, \mathbb{C}^{N}\right)$ acting on Dirac fields $\psi \in C^{\infty}\left(M, \mathbb{C}^{N}\right)$, with the classical limit $P_{(0)}:=\left.P\right|_{\lambda=0}=\not D_{(0)}+m$, where $m \in \mathbb{R}$ is a mass term. Examples are noncommutative Dirac operators in the sense of Definition 3.3 with an additional mass term, i.e. $P=\not D+m$. It is well known that for the classical massive Dirac operator $P_{(0)}=\not D_{(0)}+m: C^{\infty}\left(M, \mathbb{C}^{N}\right) \rightarrow C^{\infty}\left(M, \mathbb{C}^{N}\right)$ on globally hyperbolic spacetimes there exists a unique retarded and advanced Green's operator $G_{(0)}^{ \pm}$, see e.g. [25] for a proof employing a modern language. We remind the reader that a retarded/advanced Green's operator is a linear map $G_{(0)}^{ \pm}: C_{0}^{\infty}\left(M, \mathbb{C}^{N}\right) \rightarrow C^{\infty}\left(M, \mathbb{C}^{N}\right)$ on compactly supported functions, which satisfies the inhomogeneous equation of motion $G_{(0)}^{ \pm} \circ P_{(0)}=P_{(0)} \circ G_{(0)}^{ \pm}=$id and the support condition $\operatorname{supp}\left(G_{(0)}^{ \pm} \varphi\right) \subseteq J^{ \pm}(\operatorname{supp}(\varphi))$, for all $\varphi \in C_{0}^{\infty}\left(M, \mathbb{C}^{N}\right)$, where $J^{ \pm}(\operatorname{supp}(\varphi))$ is the forward/backward lightcone of the $\operatorname{set} \operatorname{supp}(\varphi)$. If we assume that our noncommutative Cartan geometry $(V, \Omega)$ over our twisted manifold $(M, \mathcal{F})$ is such that $V_{(0)}=\left.V\right|_{\lambda=0}$ is the vielbein of a globally hyperbolic Lorentzian metric on $M$, then we can find a unique retarded and advanced Green's operator $G^{ \pm}$for $P$. Let us write $P=\sum_{n=0}^{\infty} \lambda^{n} P_{(n)}$, then a construction as in $\left[27\right.$, Theorem 1] shows that the Green's operators $G^{ \pm}=\sum_{n=0}^{\infty} \lambda^{n} G_{(n)}^{ \pm}$for $P$ are given by, 
for $n \geq 1$,

$$
G_{(n)}^{ \pm}:=\sum_{k=1}^{n} \sum_{j_{1}=1}^{n} \cdots \sum_{j_{k}=1}^{n}(-1)^{k} \delta_{j_{1}+\cdots+j_{k}, n} G_{(0)}^{ \pm} \circ P_{\left(j_{1}\right)} \circ \cdots \circ G_{(0)}^{ \pm} \circ P_{\left(j_{k}\right)} \circ G_{(0)}^{ \pm},
$$

where $\delta_{n, m}$ is the Kronecker delta. In an earlier version of this manuscript, as well as in [27, Theorem 1], it was assumed that $P_{(n)}, n \geq 1$, are differential operators of compact support. However, due to recent advances in understanding Green-hyperbolic operators [8], this condition turns out to be unnecessary, since the compositions in (5.1) are well-defined also for generic differential operators $P_{(n)}$.

Using the Green's operators for $P$, we can characterize the solution space Sol := $\{\psi \in$ $\left.C_{\mathrm{sc}}^{\infty}\left(M, \mathbb{C}^{N}\right): P \psi=0\right\}$, where the subscript sc denotes functions of spacelike compact support. All solutions are obtained by the causal propagator $G:=G^{+}-G^{-}: C_{0}^{\infty}\left(M, \mathbb{C}^{N}\right) \rightarrow C_{\mathrm{sc}}^{\infty}\left(M, \mathbb{C}^{N}\right)$, since the following sequence of linear maps is an exact complex

$$
\{0\} \longrightarrow C_{0}^{\infty}\left(M, \mathbb{C}^{N}\right) \stackrel{P}{\longrightarrow} C_{0}^{\infty}\left(M, \mathbb{C}^{N}\right) \stackrel{G}{\longrightarrow} C_{\mathrm{sc}}^{\infty}\left(M, \mathbb{C}^{N}\right) \stackrel{P}{\longrightarrow} C_{\mathrm{sc}}^{\infty}\left(M, \mathbb{C}^{N}\right) .
$$

The proof of this statement is similar to the one of [27, Theorem 2], hence we can omit it here.

With these tools we can construct the canonical anti-commutation relation (CAR) algebra corresponding to our differential operators $P: C^{\infty}\left(M, \mathbb{C}^{N}\right) \rightarrow C^{\infty}\left(M, \mathbb{C}^{N}\right)$. This is the observable algebra of the quantized noncommutative Dirac field. For this construction we also require a hermitian inner product $\langle\cdot, \cdot\rangle$ on $C^{\infty}\left(M, \mathbb{C}^{N}\right)$ and that $P$ is formally self-adjoint. In $D=2$ and $D=4$ the inner product is given in (2.9) and (2.7), respectively. By Proposition 4.3 the Aschieri-Castellani Dirac operator is formally self-adjoint in $D=2$ and $D=4$ for $\star$-torsion free $(V, \Omega)$. The same holds true for the massive Aschieri-Castellani Dirac operator $\not^{\mathrm{AC}}+m$, which thus provides an example for a differential operator $P$ with the properties we are looking for.

For constructing the CAR algebra, let us define another inner product on $C_{0}^{\infty}\left(M, \mathbb{C}^{N}\right)$ by using the causal propagator $G=G^{+}-G^{-}$corresponding to $P$, for all $\varphi_{1}, \varphi_{2} \in C_{0}^{\infty}\left(M, \mathbb{C}^{N}\right)$,

$$
\left\langle\left\langle\varphi_{1}, \varphi_{2}\right\rangle\right\rangle:=i\left\langle\varphi_{1}, G \varphi_{2}\right\rangle .
$$

Since $\left\langle\varphi_{1}, \varphi_{2}\right\rangle^{*}=\left\langle\varphi_{2}, \varphi_{1}\right\rangle$ and $P$ is by assumption formally self-adjoint (which implies that $G$ is formally skew-adjoint) we obtain

$$
\left\langle\left\langle\varphi_{1}, \varphi_{2}\right\rangle\right\rangle^{*}=-i\left\langle G \varphi_{2}, \varphi_{1}\right\rangle=i\left\langle\varphi_{2}, G \varphi_{1}\right\rangle=\left\langle\left\langle\varphi_{2}, \varphi_{1}\right\rangle\right\rangle .
$$

Due to the exact sequence $(5.2)$ and the fact that $G$ is formally skew-adjoint, $\langle\langle\cdot, \cdot\rangle\rangle$ induces a hermitian inner product on the quotient $H:=C_{0}^{\infty}\left(M, \mathbb{C}^{N}\right) / P\left[C_{0}^{\infty}\left(M, \mathbb{C}^{N}\right)\right]$. Furthermore, the inner product $\langle\langle\cdot, \cdot\rangle\rangle$ on $H$ is positive-definite. To show this statement let us consider the classical limit $\lambda=0$. We find by using Green's formula [34, p. 160, Proposition 9.1] that the inner product in this limit is, for all $\varphi_{1},\left.\varphi_{2} \in H\right|_{\lambda=0}=C_{0}^{\infty}\left(M, \mathbb{C}^{N}\right) / P_{(0)}\left[C_{0}^{\infty}\left(M, \mathbb{C}^{N}\right)\right]$,

$$
\begin{aligned}
\left.\left\langle\left\langle\varphi_{1}, \varphi_{2}\right\rangle\right\rangle\right|_{\lambda=0} & =\left.i\left\langle P_{(0)} G_{(0)}^{ \pm} \varphi_{1}, G_{(0)} \varphi_{2}\right\rangle\right|_{\lambda=0}=i \int_{\Sigma} \overline{i \gamma_{a} n^{a} G_{(0)} \varphi_{1}} G_{(0)} \varphi_{2} \operatorname{vol}_{\Sigma} \\
& =\int_{\Sigma}\left(G_{(0)} \varphi_{1}\right)^{\dagger} G_{(0)} \varphi_{2} \operatorname{vol}_{\Sigma} .
\end{aligned}
$$

Here $\Sigma$ is any Cauchy surface and $n=E_{a} n^{a}$ its future-pointing normal vector field. In the last equality we have used that we can choose $n=E_{0}$. Since the inner product $\langle\langle\cdot, \cdot\rangle\rangle$ is positivedefinite at order $\lambda^{0}$ it is positive-definite to all orders in the deformation parameter.

The inner-product space $(H,\langle\langle\cdot, \cdot\rangle\rangle)$ can be quantized in terms of a CAR algebra, see e.g. [9] for a modern review of these techniques: To any element $\varphi \in H$ we associate an abstract 
operator $a(\varphi)$ and consider the free unital $*$-algebra $A^{\text {free }}$ generated by all $a(\varphi), \varphi \in H$. We define the CAR algebra $A^{\mathrm{CAR}}:=A^{\text {free }} / \mathcal{I}$ as the quotient of $A^{\text {free }}$ by the both-sided $*$-ideal $\mathcal{I}$ generated by the elements, for all $\varphi_{1}, \varphi_{2} \in H$ and $\alpha_{1}, \alpha_{2} \in \mathbb{C}$,

$$
\begin{aligned}
& a\left(\alpha_{1} \varphi_{1}+\alpha_{2} \varphi_{2}\right)-\alpha_{1} a\left(\varphi_{1}\right)-\alpha_{2} a\left(\varphi_{2}\right), \\
& \left\{a\left(\varphi_{1}\right), a\left(\varphi_{2}\right)\right\}, \\
& \left\{a\left(\varphi_{1}\right)^{*}, a\left(\varphi_{2}\right)\right\}-\left\langle\left\langle\varphi_{1}, \varphi_{2}\right\rangle\right\rangle 1,
\end{aligned}
$$

where $\{\cdot, \cdot\}$ is the anti-commutator. The interpretation of this quotient is as follows: (5.3a) allows us to regard $a(\psi)$ as smeared linear field operators. (5.3b) and (5.3c) encode the CAR. The on-shell condition is already implemented in $H$. In the physics literature, the Dirac field operator is typically denoted by $\Psi(x)$ and its adjoint by $\bar{\Psi}(x)$. This notation is related to ours by $a(\varphi)=\langle\Psi, \varphi\rangle$ and $a(\varphi)^{*}=\langle\varphi, \Psi\rangle$, where by the inner products we (formally) denote the smearing of the field operators by test functions. In this notation (5.3b) states that $\Psi(x)$ anticommutes with $\Psi(y)$ and (5.3c) that the anti-commutator between $\bar{\Psi}(x)$ and $\Psi(y)$ is non-trivial.

We end this section with a comparison to the more conventional approach to quantum field theory on noncommutative spacetimes as followed e.g. in $[5,7,19,35]$, where the algebra of field operators is also deformed by the twist. This is motivated by the desire for a representation of the Moyal-Weyl deformed Poincaré Hopf algebra on this algebra. While for highly symmetric models like the Moyal-Weyl Minkowski spacetime this is certainly an interesting approach, it unfortunately does not generalize to situations where there are no isometries, or where the twist is generated not by Killing vector fields alone. The relevant argument, which has been given already in the appendix of [30], is that generic vector fields on spacetime can not be represented on the algebra of field operators. Our formulation of noncommutative quantum field theory is complementary to this approach: It is valid for generic twisted curved spacetimes, but it obscures the role of twisted symmetry Hopf algebras in the very special cases where they are available.

\section{Explicit examples}

In this section we will explicitly study the noncommutative Dirac operators discussed in Section 3 on two noncommutative (curved) spacetimes. For their attractive features, e.g. as solutions to noncommutative Einstein equations, we will focus on semi-Killing deformations. As shown in Section 4, our examples of noncommutative Dirac operators coincide in this case and we will collectively denote them by $\not D$. These studies are complementing our explicit examples of deformed Klein-Gordon operators [31].

\section{$6.1 \kappa$-Minkowski spacetime}

As a first example we consider $M=\mathbb{R}^{4}$ with global coordinates denoted by $x^{\mu}=\left(t, x^{j}\right)$ and the Minkowski vierbein $V=\gamma_{a} \delta_{\mu}^{a} \mathrm{~d} x^{\mu}$, along with the spin connection $\Omega=0$. For the twist (2.1) we use $X_{1}=\partial_{t}$ and $X_{2}=x^{j} \partial_{j}$, which yields a semi-Killing twist. The commutation relations of the coordinate functions are those of $\kappa$-Minkowski spacetime, i.e. $\left[t, x^{j}\right]=i \lambda x^{j}$ and $\left[x^{i}, x^{j}\right]=0$. This example has been studied intensively in the literature, see e.g. [10, 12, 20, 22, 23, 24]. Furthermore, various fields with their equation of motion operators have been studied on this particular noncommutative spacetime, see [31] for the scalar field and [16] for the U(1) gauge field. We supplement these studies by the Dirac field with equation of motion operator given by any of the noncommutative Dirac operators introduced in Section 3, which all coincide for this model since $\mathcal{F}$ is semi-Killing. For spectral triple approaches to Dirac operators on the $\kappa$-Minkowski space we refer the reader to $[15,21]$. 
Using that $\mathcal{L}_{X_{1}}\left(V^{a}\right)=0, \mathcal{L}_{X_{2}}\left(V^{0}\right)=0$ and $\mathcal{L}_{X_{2}}\left(V^{j}\right)=V^{j}$ we obtain for the $\star$-contraction the following expression

$$
\iota_{E_{0}}^{\star}\left(\mathrm{d}_{\Omega} \psi\right)=\partial_{t} \psi, \quad \iota_{E_{j}}^{\star}\left(\mathrm{d}_{\Omega} \psi\right)=e^{\frac{i \lambda}{2} \partial_{t}} \partial_{j} \psi .
$$

Since our noncommutative Dirac operators coincide for this model we choose to calculate the simplest one, which is the contraction Dirac operator (3.4), and find

$$
\not D \psi=i \gamma^{a} \iota_{E_{a}}^{\star}\left(\mathrm{d}_{\Omega} \psi\right)=i\left(\gamma^{0} \partial_{t} \psi+\gamma^{j} e^{\frac{i \lambda}{2} \partial_{t}} \partial_{j} \psi\right) \text {. }
$$

For the solutions of the noncommutative Dirac equation $\not D \psi=0$ we can derive a dispersion relation by squaring the equation of motion operator $\not D$. More explicitly, this yields

$$
\square:=-\not D^{2}=\partial_{t}^{2}-\triangle e^{i \lambda \partial_{t}}
$$

where $\triangle:=\partial_{1}^{2}+\partial_{2}^{2}+\partial_{3}^{2}$ is the spatial Laplacian. To study the dispersion relation we make a plane wave ansatz $\psi=\chi e^{i\left(E t+k_{j} x^{j}\right)}$, where $E$ is the energy, $k_{j}$ the momentum and $\chi \in \mathbb{C}^{4}$ a polarization spinor. Since $\not D \psi=0$ implies $\square \psi=0$ we obtain the deformed energy-momentum relation

$$
E^{2}-e^{-\lambda E} \mathbf{k}^{2}=0 \quad \Leftrightarrow \quad E^{2} e^{\lambda E}=\mathbf{k}^{2} .
$$

From the equation of motion $\not D \psi=0$ we further obtain a condition on the polarization spinor

$$
\left(\gamma^{0} E+\gamma^{j} k_{j} e^{-\frac{\lambda}{2} E}\right) \chi=0 .
$$

Without loss of generality we choose the spatial momentum along the third direction, i.e. $\mathbf{k}=$ $(0,0, k)$, such that $(6.1 \mathrm{~b})$ becomes $\left(\gamma^{0} E+\gamma^{3} k e^{-\frac{\lambda}{2} E}\right) \chi=0$. Using the on-shell condition (6.1a) this becomes independent of $\lambda$ and reduces to the analogous equation in the commutative case. We thus find that the physical spin polarizations, which are characterized as the solutions of $(6.1 \mathrm{~b})$, do not receive noncommutative corrections. Hence, this type of noncommutative geometry does not introduce an anomalous spin precession.

\subsection{Noncommutative anti de Sitter space}

We now turn to a curved spacetime example, for which the natural first candidates are the maximally symmetric (anti) de Sitter ((A)dS) spacetimes. We choose AdS which is of relevance for model building in particle physics and AdS/CFT, but note that similar calculations for the cosmologically relevant dS are fully analogous. A particle-physics model employing a deformation of AdS can be found in [28].

We focus on the Poincaré patch of 4-dimensional AdS, that is, $M=\mathbb{R}^{3} \times(0, \infty)$ with coordinates $x^{\mu}=\left(x^{i}, z\right)$ and the vierbein $V=\gamma_{a} R z^{-1} \delta_{\mu}^{a} \mathrm{~d} x^{\mu}$. The generalization to higher dimensions is straightforward and just amounts to using the higher dimensional Clifford algebras. In the following we fix the radius of curvature to $R=1$, and for the gamma-matrices we denote the contraction with the (inverse) vielbein explicitly by a hat, e.g. $\hat{\gamma}^{\mu}:=E_{a}^{\mu} \gamma^{a}=z \delta_{a}^{\mu} \gamma^{a}$. To deform this space we employ the twist (2.1) with the $2 N$ mutually commuting vector fields $X_{\alpha}$, $\alpha=1, \ldots, 2 N$, given by

$$
X_{2 n-1}=T_{2 n-1}^{i} \partial_{i}, \quad X_{2 n}=\vartheta(z) T_{2 n}^{i} \partial_{i}, \quad n=1,2, \ldots, N .
$$

In this expression the $T_{\alpha}^{i}$ are real numbers and $\vartheta(z) \in C^{\infty}(0, \infty)$ is a real valued function. Notice that this twist is semi-Killing, since all $X_{2 n-1}$ are Killing vector fields. The $\star$-commutation relations of the coordinate functions $\left(x^{i}, z\right)$ read

$$
\left[x^{i}, x^{j}\right]=i \lambda \vartheta(z) \Theta^{\alpha \beta} T_{\alpha}^{i} T_{\beta}^{j}, \quad\left[x^{i}, z\right]=0 .
$$


Hence, this model describes a $z$-dependent Moyal-Weyl deformation of the $\mathbb{R}^{3}$ hypersurfaces at constant $z$. The $\star$-torsion free spin connection is $\Omega=-\frac{1}{2} V^{i} \gamma_{i 3}$.

To compute the $\star$-contraction $\iota_{E_{a}}^{\star}\left(\mathrm{d}_{\Omega} \psi\right)$ we first notice that $\mathcal{L}_{X_{2 n-1}}\left(V^{a}\right)=0, \mathcal{L}_{X_{2 n}}\left(V^{i}\right)=$ $\vartheta^{\prime}(z) T_{2 n}^{i} V^{3}$ and $\mathcal{L}_{X_{2 n}}\left(V^{3}\right)=0$. We then obtain

$$
\iota_{E_{i}}^{\star}\left(\mathrm{d}_{\Omega} \psi\right)=z \partial_{i} \psi+\frac{\gamma_{i 3}}{2} \psi, \quad \iota_{E_{3}}^{\star}\left(\mathrm{d}_{\Omega} \psi\right)=z \partial_{z} \psi+\frac{i \lambda}{2} z \vartheta^{\prime}(z) \mathcal{T} \psi,
$$

where $\mathcal{T}:=\mathcal{T}^{i j} \partial_{i} \partial_{j}:=\sum_{n=1}^{N} T_{2 n}^{i} T_{2 n-1}^{j} \partial_{i} \partial_{j}$. Since the twist is semi-Killing and our examples of noncommutative Dirac operators are therefore equivalent, we once again choose (3.4) as the technically most convenient one, and find

$$
\not D \psi=i \gamma^{a} \iota_{E_{a}}^{\star}\left(\mathrm{d}_{\Omega} \psi\right)=\not D_{(0)} \psi+\frac{\lambda}{2} z \vartheta^{\prime}(z) \gamma_{3} \mathcal{T} \psi
$$

where $\not_{(0)}$ is the classical Dirac operator. This is the equation of motion operator stemming from the action

$$
S=\langle\psi, \not D \psi\rangle=\int_{M} \bar{\psi} \not D \psi \mathrm{vol},
$$

where in the last equality we have used that for the present model the inner product (2.7) coincides with the undeformed one.

A crucial point for the construction of quantum fields on AdS is the existence of a finite inner product, which is closely related to the choice of boundary conditions, see e.g. [11] for an early reference. This issue is conveniently analyzed in terms of the hypersurface inner product on the space of solutions of the noncommutative Dirac equation, which we compute in the following. The resulting inner product space can then be quantized by following the CAR-construction outlined in Section 5. Following the strategy developed in [36] we consider variations of the action functional (6.5) and derive a conserved current. Explicitly, we obtain for the current density of two solutions $\psi_{1}$ and $\psi_{2}$

$$
i J^{\mu}=i \overline{\psi_{1}} \hat{\gamma}^{\mu} \psi_{2} \sqrt{|g|}+\delta_{i}^{\mu} \mathcal{T}^{i j} \frac{\lambda}{2} z \vartheta^{\prime}(z) \sqrt{|g|}\left(\overline{\psi_{1}} \gamma_{3} \partial_{j} \psi_{2}-\overline{\partial_{j} \psi_{1}} \gamma_{3} \psi_{2}\right),
$$

where $\sqrt{|g|}=z^{-4}$ is the square root of the metric determinant. Since $\nabla_{\mu} J^{\mu}=0$ whenever $\not D \psi_{1}=0$ and $\not D \psi_{2}=0, J^{\mu}$ is a conserved density. We integrate it over a fixed-time hypersurface $\Sigma$ with normal vector field $n^{\mu}=(1,0,0,0)^{\mu}$ to obtain the hypersurface inner product

$$
\left(\psi_{1}, \psi_{2}\right)=\int_{\Sigma}\left(\psi_{1}^{\dagger} \psi_{2}+i \mathcal{T}^{0 j} \frac{\lambda}{2} z \vartheta^{\prime}(z)\left(\overline{\partial_{j} \psi_{1}} \gamma_{3} \psi_{2}-\overline{\psi_{1}} \gamma_{3} \partial_{j} \psi_{2}\right)\right) \operatorname{vol}_{\Sigma} .
$$

That inner product is conserved only up to boundary terms, which can not be assumed to vanish on AdS. Demanding actual conservation then yields the admissible boundary conditions. A wellmotivated restriction on the deformation is to demand $\mathcal{T}^{0 i}=0$, in which case the deformation is purely in the spatial part and no higher-order time derivatives are introduced. In that case the hypersurface inner product (6.6) coincides with the undeformed one, i.e. $\left(\psi_{1}, \psi_{2}\right)=\int_{\Sigma} \psi_{1}^{\dagger} \psi_{2} \mathrm{vol}_{\Sigma}$. We would like to stress that the solutions of the noncommutative Dirac equation are still affected by the deformation, and it would hence be of interest to study the effect of different choices of $T_{\alpha}^{i}$ and $\vartheta(z)$. A natural choice would for example be such that $\mathcal{T}=\triangle$ is the spatial Laplacian on the hypersurfaces of constant $z$, as discussed in [31] for the Klein-Gordon field. This choice can be implemented by taking the vector fields (6.2) to be parallel, $T_{2 n}^{i}=T_{2 n-1}^{i}$ for all $n$. The resulting noncommutative Dirac operator (6.4) is still deformed, despite the vanishing $\star$-commutation relations of the coordinates (6.3). This shows that our noncommutative Dirac operator does not only depend on the deformed algebra of functions, but also on the deformed differential calculus, which in this case is not equal to the classical de Rham calculus (see also [32]). 


\section{A Clifford algebra conventions}

For the 4-dimensional Clifford algebra we use the gamma-matrix conventions of [1], which are

$$
\begin{array}{ll}
\eta_{a b}=\operatorname{diag}(1,-1,-1,-1)_{a b}, & \left\{\gamma_{a}, \gamma_{b}\right\}=2 \eta_{a b}, \quad \gamma_{a b}:=\frac{1}{2}\left[\gamma_{a}, \gamma_{b}\right], \\
\gamma_{5}:=i \gamma_{0} \gamma_{1} \gamma_{2} \gamma_{3}, \quad \gamma_{5}^{2}=1, \quad \epsilon_{0123}=-\epsilon^{0123}=1, \quad \gamma_{a}^{\dagger}=\gamma_{0} \gamma_{a} \gamma_{0}, \quad \gamma_{5}^{\dagger}=\gamma_{5} .
\end{array}
$$

For the $D=2$ Clifford algebra we use $\eta_{a b}=\operatorname{diag}(1,-1)_{a b}$ and the Clifford relation $\left\{\gamma_{a}, \gamma_{b}\right\}=$ $2 \eta_{a b}$ is satisfied by the $2 \times 2$-matrices

$$
\gamma_{0}=\left(\begin{array}{cc}
0 & 1 \\
1 & 0
\end{array}\right), \quad \gamma_{1}=\left(\begin{array}{cc}
0 & -1 \\
1 & 0
\end{array}\right), \quad \gamma_{3}:=\gamma_{0} \gamma_{1}
$$

We note that $\gamma_{a b}:=\frac{1}{2}\left[\gamma_{a}, \gamma_{b}\right]=\epsilon_{a b} \gamma_{3}$, where $\epsilon_{a b}$ is the 2-dimensional $\epsilon$-tensor with $\epsilon_{01}=1$. We further have $\gamma_{a}^{\dagger}=\gamma_{0} \gamma_{a} \gamma_{0}, \gamma_{3}^{\dagger}=\gamma_{3}, \gamma_{3}^{2}=1$ and $\gamma_{3} \gamma_{a}=-\gamma_{a} \gamma_{3}$.

\section{Acknowledgements}

We would like to thank the referees for their constructive and useful comments. CFU is supported by Deutsche Forschungsgemeinschaft through the Research Training Group GRK 1147 Theoretical Astrophysics and Particle Physics.

\section{References}

[1] Aschieri P., Castellani L., Noncommutative $D=4$ gravity coupled to fermions, J. High Energy Phys. 2009 (2009), no. 6, 086, 18 pages, arXiv:0902.3817.

[2] Aschieri P., Castellani L., Noncommutative gravity solutions, J. Geom. Phys. 60 (2010), 375-393, arXiv:0906.2774.

[3] Aschieri P., Castellani L., Noncommutative gravity coupled to fermions: second order expansion via SeibergWitten map, J. High Energy Phys. 2012 (2012), no. 7, 184, 27 pages, arXiv:1111.4822.

[4] Aschieri P., Dimitrijević M., Meyer F., Wess J., Noncommutative geometry and gravity, Classical Quantum Gravity 23 (2006), 1883-1911, hep-th/0510059.

[5] Aschieri P., Lizzi F., Vitale P., Twisting all the way: from classical mechanics to quantum fields, Phys. Rev. D 77 (2008), 025037, 16 pages, arXiv:0708.3002.

[6] Aschieri P., Schenkel A., Noncommutative connections on bimodules and Drinfeld twist deformation, arXiv:1210.0241.

[7] Balachandran A.P., Pinzul A., Qureshi B.A., Twisted Poincaré invariant quantum field theories, Phys. Rev. D 77 (2008), 025021, 9 pages, arXiv:0708.1779.

[8] Bär C., Green-hyperbolic operators on globally hyperbolic spacetimes, arXiv:1310.0738.

[9] Bär C., Ginoux N., Classical and quantum fields on Lorentzian manifolds, in Global Differential Geometry, Springer Proceedings in Mathematics, Vol. 17, Editors C. Bär, J. Lohkamp, M. Schwarz, Springer, Berlin, 2012, 359-400, arXiv:1104.1158.

[10] Borowiec A., Pachol A., $\kappa$-Minkowski spacetime as the result of Jordanian twist deformation, Phys. Rev. D 79 (2009), 045012, 11 pages, arXiv:0812.0576.

[11] Breitenlohner P., Freedman D.Z., Stability in gauged extended supergravity, Ann. Physics 144 (1982), 249-281.

[12] Bu J.-G., Kim H.-C., Lee Y., Vac C.H., Yee J.H., $\kappa$-deformed spacetime from twist, Phys. Lett. B 665 (2008), 95-99, hep-th/0611175.

[13] Connes A., Noncommutative geometry, Academic Press Inc., San Diego, CA, 1994.

[14] Connes A., Landi G., Noncommutative manifolds, the instanton algebra and isospectral deformations, Comm. Math. Phys. 221 (2001), 141-159, math.QA/0011194. 
[15] D'Andrea F., Spectral geometry of $\kappa$-Minkowski space, J. Math. Phys. 47 (2006), 062105, 19 pages, hep-th/0503012.

[16] Dimitrijević M., Jonke L., A twisted look on kappa-Minkowski: U(1) gauge theory, J. High Energy Phys. 2011 (2011), no. 12, 080, 23 pages, arXiv:1107.3475.

[17] Doplicher S., Fredenhagen K., Roberts J.E., Spacetime quantization induced by classical gravity, Phys. Lett. B 331 (1994), 39-44.

[18] Doplicher S., Fredenhagen K., Roberts J.E., The quantum structure of spacetime at the Planck scale and quantum fields, Comm. Math. Phys. 172 (1995), 187-220, hep-th/0303037.

[19] Fiore G., Wess J., Full twisted Poincaré symmetry and quantum field theory on Moyal-Weyl spaces, Phys. Rev. D 75 (2007), 105022, 13 pages, hep-th/0701078.

[20] Govindarajan T.R., Gupta K.S., Harikumar E., Meljanac S., Meljanac D., Twisted statistics in $\kappa$-Minkowski spacetime, Phys. Rev. D 77 (2008), 105010, 6 pages, arXiv:0802.1576.

[21] Iochum B., Masson T., Schücker T., Sitarz A., Compact $\kappa$-deformation and spectral triples, Rep. Math. Phys. 68 (2011), 37-64, arXiv:1004.4190.

[22] Jurić T., Meljanac S., Štrajn R., Differential forms and $\kappa$-Minkowski spacetime from extended twist, Eur. Phys. J. C 73 (2013), 2472-2480, arXiv:1211.6612.

[23] Kim H.-C., Lee Y., Rim C., Yee J.H., Differential structure on the $\kappa$-Minkowski spacetime from twist, Phys. Lett. B 671 (2009), 398-401, arXiv:0808.2866.

[24] Meljanac S., Samsarov A., Štrajn R., $\kappa$-deformation of phase space; generalized Poincaré algebras and R-matrix, J. High Energy Phys. 2012 (2012), no. 8, 127, 16 pages, arXiv:1204.4324.

[25] Mühlhoff R., Cauchy problem and Green's functions for first order differential operators and algebraic quantization, J. Math. Phys. 52 (2011), 022303, 7 pages, arXiv:1001.4091.

[26] Ohl T., Schenkel A., Cosmological and black hole spacetimes in twisted noncommutative gravity, J. High Energy Phys. 2009 (2009), no. 10, 052, 12 pages, arXiv:0906.2730.

[27] Ohl T., Schenkel A., Algebraic approach to quantum field theory on a class of noncommutative curved spacetimes, Gen. Relativity Gravitation 42 (2010), 2785-2798, arXiv:0912.2252.

[28] Ohl T., Schenkel A., Uhlemann C.F., Spacetime noncommutativity in models with warped extradimensions, J. High Energy Phys. 2010 (2010), no. 7, 029, 16 pages, arXiv:1002.2884.

[29] Schenkel A., Noncommutative gravity and quantum field theory on noncommutative curved spacetimes, Ph.D. thesis, University of Würzburg, 2011, available at http://opus.bibliothek.uni-wuerzburg.de/ volltexte/2011/6582/, arXiv:1210.1115.

[30] Schenkel A., QFT on homothetic Killing twist deformed curved spacetimes, Gen. Relativity Gravitation 43 (2011), 2605-2630, arXiv:1009.1090.

[31] Schenkel A., Uhlemann C.F., Field theory on curved noncommutative spacetimes, SIGMA 6 (2010), 061, 19 pages, arXiv:1003.3190.

[32] Schenkel A., Uhlemann C.F., High energy improved scalar quantum field theory from noncommutative geometry without UV/IR-mixing, Phys. Lett. B 694 (2010), 258-260, arXiv:1002.4191.

[33] Schupp P., Solodukhin S., Exact black hole solutions in noncommutative gravity, arXiv:0906.2724.

[34] Taylor M.E., Partial differential equations. I. Basic theory, Applied Mathematical Sciences, Vol. 115, Springer-Verlag, New York, 1996.

[35] Zahn J., Remarks on twisted noncommutative quantum field theory, Phys. Rev. D 73 (2006), 105005, 6 pages, hep-th/0603231.

[36] Zuckerman G.J., Action principles and global geometry, in Mathematical Aspects of String Theory (San Diego, Calif., 1986), Adv. Ser. Math. Phys., Vol. 1, World Sci. Publishing, Singapore, 1987, 259-284. 